der Gefahr, auch solche Phänomene und Prozesse kurzerhand zu „kulturellen“ zu erklären, die zunächst in gesellschaftlichen und politischen Ursachen begründet sind. Es ist eine Gefahr der „Kulturalisierung“ unseres Blickes auf die Gesellschaft, einer möglicherweise zu starken Fixierung auf die Zeichen und die Formen menschlichen Handelns zu Lasten der politischen Motive und der sozialen Ursachen. So verkörpern sich im Geschlechterverhältnis eben nicht nur kulturelle Praxen, sondern auch gesellschaftspolitische Macht- und Herrschaftsmittel. Ausländerfeindlichkeit läßt sich nicht nur als kulturelle Abgrenzungsstrategie erklären, sondern muß in ihren sozialen Ursachen und politischen Zielsetzungen zurückverfolgt werden, um nicht bei der simplen Formel zu enden, sie sei lediglich eine mentale Reaktion auf ein kulturelles „Anderssein“. Die Selbstdarstellung als „ethnische“ Gruppe bedeutet auch ein Stück kultureller Inszenierung, durch die Anerkennung, Rechte und Mittel eingefordert werden; und kulturelles „Anderssein“ fußt sehr oft auf sozialen Lagerunterschieden, in denen sich ungleiche materielle Lebenschancen ausdrücken - nicht einfach unterschiedliche Lebensstile, wie gern suggeriert wird. Mit anderen Worten: Die gewünschte Nähe des Kulturund Alltagsbegriffs zur Praxis der Menschen bedeutet auch eine hohe theoretische und methodologische Herausforderung, weil sie deutlich macht, wie eng Kultur und Gesellschaft, wie eng aber auch Gesellschaftspraxis und Wissenschaftspraxis ineinander verwoben sind (Kaschuba I995b). Als Alltagsmenschen teilen wir vielfach jene zeitgeisthaft praktizierten Kultur- und Politikauffassungen, die wir als WissenschaftlerInnen kritisch und distanziert beleuchten wollen.

\title{
2. Konstruktionen: Identität und Ethnizität
}

Man kann diese Feststellungen gleich auf die beiden Begriffe ausdehnen, die nun unter dem Stichwort „Konstruktionen“ vorgestellt werden. Denn diese Charakterisierung soll darauf hinweisen, daß Vorstellungen von sozialer oder ethnischer Identität sich stets an Bilder knüpfen, in denen unbewußte wie bewußte Anteile enthalten sind: Einerseits wird damit ein umstandsloses So-Sein praktiziert und gelebt, andererseits ein absichtsvolles So-Sein/So-Erscheinen-Wollen beschrieben. Es sind stets zugleich fast reflexhafte, alltägliche Selbstverständlichkeiten und Standorte, die unser Identisch-Sein mit uns ausmachen, aber eben auch strategische Setzungen und Selbstverständnisse, die bewußte Effekte unserer Selbstdarstellung und unserer Wahrnehmung durch andere erzeugen sollen. Ethnische Identitätsvorstellungen spiegeln diese zweite Seite der „Konstruktion“ vielleicht am auffäligsten wider. Sie werden deshalb hier als ein eigenes 
Modell von Identitätsentwürfen genannt, obwohl sie eigentlich nur eine von vielen Varianten des allgemeinen Identitätsbegriffs bilden.

\section{Kollektive Identitäten}

Ähnlich wie Kultur und Alltag verkörpert Identität einen Begriff, der seit mindestens zwei, drei Jahrzehnten als Schlagwort in sozialwissenschaftlichen wie politischen Diskussionen vielfältig präsent ist und deswegen nicht nur geschätzt wird. Schon im Jahr I978 kommentierte Hermann Bausinger diese Ambivalenz mit den Sätzen: „Zweifellos ist Identität ein modischer Begriff; aber sprachliche Moden - auch solche der Wissenschaftssprachen - kommen nicht von ungefähr. Von Identität ist deshalb so viel die Rede, weil Identität zum Problem geworden ist. Der Begriff verkörpert, so weit die Konnotationen im einzelnen auseinanderlaufen mögen, ein Moment von Ordnung und Sicherheit inmitten des Wechsels; und sein besonderer Reiz liegt dabei darin, daß er nicht eigentlich die Bedeutung von Starrheit oder Erstarrung vermittelt, sondern daß er verhältnismäßig elastisch etwas Bleibendes in wechselnden Konstellationen anvisiert.“ (Bausinger 1978: 204) Dieses Schillernde des Begriffs und seiner Anwendungen produziert Anziehung wie Skepsis, jedenfalls bleibt bislang die Einsicht, daß offenbar kein anderer Begriff in ähnlich eindringlicher Weise die beständige gesellschaftliche Suche nach kulturellen Mustern innerer wie äußerer Übereinstimmung zu bezeichnen vermag.

Begriffsgeschichtlich gehört die lateinische identitas in den Bereich der Logik und beschreibt dort die Übereinstimmung eines Gegenstandes mit sich selbst, seine Einzigartigkeit, sein „In-Sich-GefestigtSein". Damit deutet sich bereits auf der etymologischen Ebene an, daß der Begriff in sich schon eine theoretische Vorannahme enthält, nämlich die Vorstellung, daß sich zwischen der Beschreibung eines Soll- und eines Ist-Zustandes jeweils Unterschiede, Defizite auftun, die nach Verringerung, nach wachsender „Identität“ drängen. Die moderne Psychologie hat diesen Begriff deshalb übernommen, um damit als ein wesentliches Ziel menschlichen und gesellschaftlichen Lebens das Streben nach Übereinstimmung mit sich selbst zu beschreiben: den Wunsch zu werden, was man ,ist“. Namentlich der schwedische Psychologe Erik Erikson (1957) hat den Aufbau eines Selbstbildes bei Kindern und Jugendlichen, das über Nachahmung der und zugleich Abgrenzung von den Erwachsenen stattfindet, in diesem Sinne charakterisiert. Er beschreibt diesen kindlichen Identitätsaufbau als einen langwierigen Prozeß sozialer Selbstfindung, der sich räumlich, körperlich, psychisch, emotional und sozial vollzieht und zunächst in der Lösung des Kindes aus der emotionalen Symbiose 
mit Eltern und Familie und in der Integration in die Gruppen Gleichaltriger besteht, der aber auch im späteren Lebenszyklus immer wieder neu zu justieren ist. Bereits in diesem Anwendungszusammenhang verweist der Begriff über eine psychologische Dimension hinaus auf kulturelle Prozesse, die in der Sozialisations- und Enkulturationsphase von Kindern und Jugendlichen sowohl die Übernahme anderer als auch die Entwicklung eigener Verhaltensmuster bezeichnen, also das Einleben in soziale Situationen und Zusammenhänge, das Einüben von deren kulturellen Regeln und Praktiken, die Übernahme von ethisch-moralischen Grundsätzen etwa religiösen Denkens bis in den Bereich der Höflichkeit oder der Eßkultur.

Insofern läßt sich der Begriff Identität als ein anthropogenes, also menschheitsgeschichtliches Grundmuster verstehen, das in den Wunsch mündet, sich als soziales Wesen in den Zusammenhang seiner Umwelt einzupassen und dabei durch Übereinstimmung wie durch Abgrenzung seinen spezifischen ,sozialen Ort“ zu finden. In diesem erweiterten Bedeutungssinn meint Identität in den Gesellschafts- und Kulturwissenschaften die Kennzeichnung eines Bildes und eines Prozesses zugleich: die Vorstellung eines sozialen So-Seins wie den Vorgang der gesellschaftlichen Aushandlung dieser Vorstellung. Diese Vorstellung wie die Aushandlung enthalten dabei immer sowohl eher feste, indisponible als auch mehr flüssige, verhandelbare Komponenten. So werden im gesellschaftlichen Alltag einerseits Feststellungen etwa geschlechtlicher, sozialer oder religiöser Identität nur selten verändert werden können, wenn es denn überhaupt gewollt ist. Andererseits sind bestimmte Wertvorstellungen, ästhetische Stile oder altersbezogene Rollen zwar immer unabdingbare Festlegungen für den Moment, um sich ein geschlossenes Selbstbild zu erhalten, ihre Inhalte jedoch sind zeitlich wandelbar, ja mitunter sogar relativ kurzwelligen Veränderungsprozessen unterworfen, wenn beispielsweise eine Alters- oder Ausbildungsschwelle überschritten wird, sich also der soziale Lebenskontext und dessen Logik verändern.

Zugleich meint Identität immer sowohl eine Ich- als auch eine Wir-Identität, zwei sich ineinander verschränkende Bedeutungsdimensionen von Selbstsein und Dazugehören. Individuelle und kollektive Identitätsvorstellungen sind zwar nicht ,,identisch“, aber sie gehen immer wieder Hand in Hand. Sie beschreiben unterschiedliche Wege der Suche nach Übereinstimmungen wie Grenzziehungen und spielen sich damit wesentlich auch in einer kulturellen Dimension der Symbole und Gesten ab, die als Kodeformeln sozialer Wechselbeziehungen und Verständigungsprozesse fungieren: Blicke wie Grußformeln, Lifestyle-T-Shirts wie nationale Embleme am Auto. Was Identität jedoch als Umschreibung eines „Bei-Sich-Seins“ letzt- 
lich bedeutet, ist zwar durch die ,groben“ Verhaltensregeln und Identitätsmerkmale generell vorbestimmt, als ein konkretes SichVerhalten $\mathrm{zu}$ sich selbst und $\mathrm{zu}$ anderen indessen unterliegt es stets ausgesprochen ,feinen“ situativen Prägungen. Wir werden in einer beliebigen Gesprächssituation zwar kaum unsere geschlechtliche oder Altersidentität grundsätzlich in Frage stellen. Wie wir darin die Züge unseres Selbstbildes jedoch nuancieren, hängt vom sozialen Kontext der Verhaltenssituation ab, also davon, welche konkreten Integrations- oder Exklusionsimpulse uns die anderen Teilnehmer dieser Situation vermitteln und über welchen Darstellungsspielraum wir dabei verfügen. Identität bezieht sich so immer auch auf den Vorgang eines konkreten Aushandelns in konkreten Situationen, in denen jeweils andere Zuordnungen und Bezüge gegeben sind: Jeder soziale Ort weist seine eigene Struktur von festliegenden Verhaltensregeln wie offenen Verhaltensspielräumen auf, die respektiert und gestaltet werden müssen. So kann Identität nur als soziale Praxis verstanden werden: als ein Umsetzen allgemeiner Regeln und Vorstellungen des eigenen So-Seins in konkretes kommunikatives und interaktives Verhalten, das sich mit jeder Veränderung der Situation wiederum selbst verändert.

Ein einprägsames Beispiel für dieses situative Spiel von Identitäten und dessen Regeln gibt der Ethnologe Werner Schiffauer im Rahmen einer Migrationsstudie, in der er aus einem Bericht über eine Feldforschung in einem türkischen Dorf zitiert: „Ein junges Ehepaar sitzt mit der Ethnologin in einem Raum. Beide necken sich und ziehen sich gegenseitig auf. Die Stimmung ist freundlich und entspannt. Sie schlägt jedoch in dem Augenblick um, in dem der Vater des Ehegatten, der Haushaltsvorstand, den Raum betritt. Plötzlich ist die Atmosphäre gespannt. Der junge Mann gibt seiner Frau knappe Befehle, die sie ohne Widerspruch ausführt." Daran schließt sich folgende Interpretation an: „Die Interaktion zwischen zwei Personen ist abhängig von Dritten. Es wird in dieser Gesellschaft nicht erwartet, daß man dem anderen immer in der gleichen Haltung gegenübertritt - im Gegenteil: In der hier beschriebenen Szene wäre es als massive Respektlosigkeit ... gegenüber dem Vater erschienen, wenn der Ehemann weiterhin Zärtlichkeit gegenüber seiner Frau gezeigt hätte. Die abrupte Verhaltensänderung des Ehemanns wirft indessen keine Probleme der Kontinuität auf, weil in diesem sozialen Universum des Dorfes jedem bewußt ist, wie die Status sich einander zuordnen, d.h. wie jeder zu jedem steht. Dies läßt sich verallgemeinern: Identität muß in der türkischen dörflichen Gesellschaft nicht vom Individuum selbst hergestellt werden, sondern sie wird gleichsam außerhalb des einzelnen durch die Transparenz des sozialen Rahmens gestiftet." (Schiffauer I99I: 47) 
Dieses Beispiel stammt aus einer in ihren sozialen Raum- und Rollenordnungen besonders fest und konservativ strukturierten Gesellschaft, die wenig individuelle Gestaltungsmöglichkeiten läßt. Dafür zeigt es um so klarer, wie eine wesentliche Grenze dieses Spielraumes dadurch festgelegt wird, daß unsere individuelle immer auch kollektive Identität ist, daß sie stets auch die Sicht Zweiter und Dritter einschließt, also aus Interaktionen wie aus „Fremdzuschreibungen" besteht. Andere sehen uns, machen sich ihr Bild von uns, „,identifizieren“ uns - als Deutsche, als religiöse Menschen oder als Individualisten. Mit diesen Zuschreibungen verbinden sie Bilder, auf deren Inhalt wir zunächst wenig Einfluß haben, die wir - wenn wir sie als negativ für uns empfinden - höchstens in der Interaktion selbst zu beeinflussen versuchen können. Wie erfolgreich, hängt davon ab, wie sehr uns dieses Bild von uns beeindruckt, wie sehr wir davon abhängig sind oder uns davon unabhängig machen.

Dieser Aushandlungsprozeß zwischen den Selbstbildern und den Fremdbildern in sozialen Begegnungs- und Beziehungssituationen gehört sicherlich zu den kompliziertesten Balanceakten, die wir im Alltag vollführen. Zugleich sind es die vielleicht wichtigsten Akte unserer Identitätsbehauptung, weil dieses „Echo“ auf uns in Gesprächen und Begegnungen für unsere psychische wie soziale Verfassung existentiell ist. Wer ohne solches Echo, ohne soziale Berührungen durch die anderen lebt, hat gleichsam seine Haut verloren, die ihn mit der Umwelt verbindet und ihn zugleich gegen diese abgrenzt.

\section{„Wieviel Heimat braucht der Mensch?“}

Wie sensibel und lebensnotwendig diese ,kulturelle Haut“ der Identität ist, zeigt sich sowohl in der Situation des sozialen Identitätsverlusts in existentiellen oder psychischen Krisen als auch in der Gefahr der sozialen Überidentifikation, wenn wir uns von einem zentralen Identitätsbezug völlig abhängig machen - etwa von einem bestimmten Körperlichkeitsbild, von der Akzeptanz einer bestimmten Bezugsgruppe, wie das in Bereichen der Jugendkultur häufig geschieht, oder von einer nationalistischen Einstellung, die sich aggressiv gegen das „Fremde“ wendet. Erfahrungen des Identitätsverlusts wie der Überidentifikation beschreiben also pathogene Situationen, die im sozialen wie im psychosomatischen Sinne krank machen können.

Im Blick auf das Individuum meint der Begriff der Identitätskrise also die intensiv erlebte Erfahrung grundlegender sozialer und kultureller Dissonanzen mit der gesellschaftlichen Umwelt. Er wird in den gegenwärtigen Diskussionen einer „reflexiven“ Moderne aber auch als Kennzeichnung gesellschaftlicher Befunde benutzt: gesellschaftliche Identitätskrisen als Ausdruck überwältigender Erfahrungen eines 
beschleunigten sozialen und kulturellen Wandels, einer globalen Veränderung ökonomischer und technologischer Rationalität, einer wachsenden sozialen Entwurzelung durch Mobilität und Migration. Es sind Bilder eines aufbrechenden und fragmentierten Gegenwartshorizonts, die heute als Beschreibung der Krise der Moderne entworfen werden - Bilder, in denen Motive der permanenten Veränderung, der politischen Unruhe, der sozialen Unsicherheit, des Verlustes kultureller Bindung dominieren - skeptische, ja bedrohliche Bilder, in denen die künftige Integrationskraft der bisherigen gesellschaftlichen Identitätsentwürfe zu Recht angezweifelt wird: Nation, Schicht, Region, Generation scheinen als soziale Bezugssysteme in globalen Horizonten zu verschwinden.

Freilich sind es keine ganz neuen Bilder. Denn viele dieser Motive gehören zu einem festen Themenkreis, der in der Wissenschaft wie in der Kunst und der Literatur der letzten hundert Jahre immer wieder aufscheint, beschrieben als ein „Unbehagen an der Moderne“, als ein grundlegendes Unsicherwerden der Gesellschaft an sich selbst. Auch damals waren die Krisenbefunde stets verbunden mit der Frage nach Konzepten der Krisenbewältigung, nach Instanzen einer neuen Identitäts- und Sinnstiftung, die man sich nicht zuletzt im Bereich der Wissenschaften erhoffte. ${ }^{22}$ Solche versichernden Gegenrezepte versuchte in der Vergangenheit gerade auch die Volkskunde zu liefern, wenn sie mit Begriffen wie Volk, Tradition, Region oder Heimat ihre rückwärtsgewandten Visionen von Ursprung und Gemeinschaft entwarf, die der Gegenwart als Haltegriffe vor einem Abgleiten in den Abgrund der Moderne dienen, ihr also wieder „Identität" bieten sollten - häufig genug in nationalistischen und ethnozentrischen Bildern. ${ }^{23}$

Wie wenig allerdings solche ideologischen Haltegriffe von „Volk“ und „Heimat" meist taugten, zeigen die Fachgeschichte wie die deutsche Geschichte insgesamt. Der Schriftsteller Jean Améry hat die Bodenlosigkeit solchen vermeintlichen Haltes in der deutschen Vergangenheit und Gegenwart immer wieder zum Thema seines literarischen Nachdenkens gemacht und diese Erfahrung auch in einem Essay mit dem Titel 〈Wieviel Heimat braucht der Mensch?〉 autobiographisch festgehalten. Darin schildert er, wie er als österreichischer Jude und deutscher Linker im Jahr I938 vor dem Nazismus nach Belgien flieht, in Antwerpen als Exilant und Antifaschist jenes Deutschland bekämpft, sich zugleich aber auch vor Heimweh nach ihm verzehrt. Nach der Besetzung Belgiens durch die Wehrmacht beteiligt sich Améry am aktiven Widerstand, wird dann I943 verhaftet und in ein Konzentrationslager gebracht. Kurz zuvor jedoch erlebt er, wie seine Wohnung, die auch als Stützpunkt der illegalen Arbeit dient, von einem im Hause wohnenden SS-Mann betreten wird, 
der sich nichtsahnend lediglich wegen des Lärms aus dieser Nachbarwohnung beschweren und seine Nachtruhe einfordern will. Die Situation wird für Améry grotesk: „Er stellte seine Forderung - und dies war für mich das eigentlich Erschreckende an der Szene - im Dialekt meiner engeren Heimat. Ich hatte lange diesen Tonfall nicht mehr vernommen, und darum regte sich in mir der aberwitzige Wunsch, ihm in seiner Mundart zu antworten. Ich befand mich in einem paradoxen, beinahe perversen Gefühlszustand von schlotternder Angst und gleichzeitig aufwallender familiärer Herzlichkeit, denn der Kerl ... erschien mir plötzlich als ein potentieller Kamerad." (Améry 1980: 85f.)

Einerseits fühlt Améry sich überwältigt durch die Rührung, diesen seit Jahren nicht mehr vernommenen Dialekt als „Heimatklang““ wieder zu hören - die Sprache als den symbolischen Ort der Heimat. Andererseits überwältigt ihn die Todesangst dieser Situation, in der sein Landsmann zu seinem Mörder werden könnte. Es ist ein fast absurder Zwiespalt, der gefühlsmäßige Momente eines völligen Identisch-Seins mit dem klaren Wissen eines absoluten Nicht-IdentischSeins verbindet. Was heißt da Heimat, was nationale Identität, wenn er bei Fremden in Belgien Sicherheit finden, während er vom Nachbarn den Tod erwarten kann? Améry antwortet darauf: „Die Feindheimat wurde von uns vernichtet, und zugleich tilgten wir das Stück eigenen Lebens aus, das mit ihr verbunden war. Der mit Selbsthaß gekoppelte Heimathaß tat wehe, und der Schmerz steigerte sich aufs unerträglichste, wenn mitten in der angestrengten Arbeit der Selbstvernichtung dann und wann auch das traditionelle Heimweh aufwallte und Platz verlangte." (Améry I980: 88)

Diese tückische Erfahrung des existentiellen Sicherheitsverlusts in einst versichernd beschriebenen und geglaubten Identitätsbildern beschränkt sich nicht auf die deutsche Geschichte wie auf Geschichte überhaupt. Sie begegnet uns gegenwärtig in zahllosen Erfahrungsberichten aus dem Krieg und den „ethnischen Säuberungen“ der letzten Jahre im ehemaligen Jugoslawien ebenso wieder wie in Erlebnissen deutscher Ausländerfeindlichkeit durch Migranten. Und sie stellt in der Tat immer wieder die Frage neu, ,wieviel Heimat" und welche Identität wir brauchen, wenn wir nicht bloß über versichernde Bilder von uns verfügen, sondern in sicheren menschlichen Beziehungen leben wollen.

Aus all dem wird deutlich, daß Identität also nichts „,ür sich“ Existentes ist, sondern stets auf soziale Relationen und kulturelle Interpretationen verweist; sie konstituiert sich überhaupt erst durch die Bezugnahme auf ein Anderes. Die klassische Konstruktion jener Bilder vom „Eigenen“ und „Fremden“ versuchte aus dieser Abgrenzung von einem Anderen als fremdem Gegenüber eine versichernde 
Wirkung zu erzielen: Das Andere konnte als „Fremdes“ getrennt vom „Eigenen“ gedacht werden, als eine „Welt da draußen“. Heute sind diese Trennungen als stimmige soziale Weltbilder, als kollektive Vorstellungen sicherlich ins Wanken geraten, weil Eigenes auch fremd und Fremdes uns vertraut geworden ist. Unsere spätmodernen Arbeits- und Lebenswelten, unser Medienkonsum und unser Reisen öffnen und verschränken unsere Wahrnehmungshorizonte. Sie lassen uns in sich überschneidenden und überlagernden Identitäten $\mathrm{zu}$ Hause sein, die vielfältiger, aber auch instabiler werden, deren versichernde Wirkungen sich räumlich wie zeitlich einschränken. Wir selbst scheinen vielfach Fremde in einem Eigenen zu werden, das sich durch soziale Differenzierung und Mobilität, durch Individualisierung und Ästhetisierung zunehmend auflöst, jedenfalls keine Erwartungen „,totaler“ Übereinstimmung mehr zuläßt (Kristeva I990; Giesen I99I). „Moderne Gesellschaft kann nur deshalb Fremdheit generalisieren, weil sie gleichzeitig Fremdheit als Sonderstatus dadurch aufhebt, daß alle Fremde sind. Paradox formuliert: Nur dort können alle Fremde sein, wo es keine Fremden mehr gibt.“ (Hahn I994: I62)

Dies macht einsichtig, weshalb es wenig hilfreich ist, von der Identität $\mathrm{zu}$ sprechen, weshalb dieser Begriff auch kaum zur systematischen Kennzeichnung eines Forschungskonzeptes taugt. Seine Nützlichkeit kann sich nur in konkreten Untersuchungsfeldern erweisen, um vor allem dort, wo Eigen- und Fremdbilder entworfen und benutzt werden, zu klären, wie diese kulturell aufgebaut und legitimiert sind und welche politisch-strategische Bedeutung ihnen ,als Diskursformationen" (Assman I993: 240) zukommt. Denn die vielgeführte Rede von der „kulturellen Identität“ erweist sich heute auf den zweiten Blick zumeist als eine überaus politische Rede.

\section{Das „ethnische Paradigma“ als Identitätskonzept}

Ein derartiger politischer - man könnte auch sagen: „kulturalistischer", also den kulturellen Aspekt absolut setzender - Verwendungszusammenhang kennzeichnet heute sicherlich den Begriff der Ethnizität. Zugleich beschreibt dieser ein besonders traditions- und folgenreiches kollektives Identitätskonzept, das aufs engste mit der Fachgeschichte der Volks- und Völkerkunde verbunden ist. Da von ihm bereits vielfach die Rede war, kann ich mich hier auf wesentliche Gesichtpunkte beschränken.

Begriffsgeschichtlich greift die Vorstellung ethnischer Identität zurück auf das griechische Wort ethnos, das im Sprachgebrauch der Antike die Gesamtheit aller nichtgriechischen Bevölkerungsgruppen bezeichnete. Es setzt semantisch also bereits ein Bewußtsein kulturel- 
ler Zusammengehörigkeit voraus, das sich aus der Wahrnehmung der „Andersartigkeit" aller anderen speist. Dies ist in doppelter Hinsicht bemerkenswert: Zum einen ist darin bereits jenes Relationale des späteren ethnischen Verständnisses in der Völker- und Volkskunde angelegt, die solche Wir-Identitäten ja als Resultat einer quasi naturwüchsigen Vergleichs- und Differenzbestimmung ,zum Anderen“ betrachten. Zum andern ist in diesem ethnos bereits eine „Feststellung von Fremdartigkeit" enthalten, die auf der Ebene des Kulturellen argumentiert: in Vorstellungen einer als ,kulturelle Daseinsform“ gedachten kollektiven Identität (Rudolph I992: 59f.).

Daraus wird freilich auch ersichtlich, daß ethnos mit der Übertragung in den deutschen Begriff des „Volkes“ später eine deutlich abweichende semantische Bedeutungsrichtung erhalten hat. Eine eigene Richtung, die nicht mehr primär auf das kulturelle Prinzip von Zusammengehörigkeitsgefühlen verweist, sondern auf das biologische Abstammungsprinzip. Volkszugehörigkeit wird in diesem Sinne als Resultat des Hineingeboren-Werdens in eine „Abstammungsgemeinschaft" verstanden. Sie meint eine blutsmäßige Bindung, die entsprechende Zuschreibungen physischer, mentaler und kultureller Verwandtschaft nach sich zieht, auch auf ,angestammte“ räumliche Grenzen und Territorien verweist und die nun als ein scheinbar ,natürliches“ Grundprinzip kollektiver Identität betrachtet wird: das Volk als Abstammungs- und Territorialgemeinschaft.

Diese Bedeutungsverschiebung vollzieht sich zu Beginn des I9. Jahrhunderts, und sie beschreibt die „Erfindung des ethnischen Paradigmas". Es ist die Zeit des Umbruchs in die Moderne, jene Epoche also, in der sich verstärkt regionale und nationale Bewegungen in Europa gegen Feudal- wie Fremdherrschaften erheben, in der zugleich das Konzept der modernen Nationalstaaten die europäische Landkarte grundlegend zu verändern beginnt und in der schließlich im Gefolge der Aufklärung neues wissenschaftliches wie pädagogisches Denken in die lokalen Wissensordnungen des Alltags eindringt. ${ }^{24}$ Damit brechen die tradierten Erfahrungshorizonte auf, alte Auffassungen von lokaler Tradition und Geschichte, von Landschaft und Raum müssen in neue, unvertraute Begriffe sozialer und staatlicher Existenz übertragen werden. So entsteht in den sozialen Lebenswelten ein mentales wie emotionales Vakuum, das durch neue Bindungsangebote gefüllt werden soll: durch die ,wärmenden“, Nähe und Gemeinschaft versprechenden Formeln von Heimat und Volk, also von einer „Volks-Kunde“, die als literarisch-ästhetisches Modell wie als ethnisches Denkprinzip wirkt und die verspricht, $\mathrm{Na}-$ tur und Kultur wieder im Ursprungsgedanken zu versöhnen. „Die behauptete Abstammungsgemeinschaft imitiert eine Familienbeziehung und einen exklusiven Gemeinsamkeitsglauben. Diese moderne 
Verwandtschaft der Ethnie wird zur geglaubten Wirklichkeit, indem der Ideologie eine emotionale Struktur eingezogen wird, die eine neue, nationale Erfahrung ermöglicht. Krieg, aber auch Geschichte, Literatur und Volksdichtung werden zu Verständigungsmustern der Ethnien, weil in ihnen Gemeinsamkeit erfahrbar wird. Das Ethnische ist eine Antwort auf die Auflösung der traditionalen Strukturen. Die Ethnien sind aber nicht naturgewachsen, sondern sie sind im intellektuellen Diskurs im Verlauf der Modernisierung hergestellt worden.“ (Köstlin I994: Iof.) An dieser „Erfindung“ sind Volkskunde und Völkerkunde in jener Zeit maßgeblich beteiligt.

Dies bedeutet nicht, daß die frühen Volks- und Völkerkundler das ethnische Denken selbst „erfunden“ haben. Wie die Etymologie des Wortes ethnos bestätigt, finden sich kulturelle Zuordnungen nach Stämmen, die zugleich oft auch Territorial- und Abstammungsverbände beschreiben sollten, bereits in der antiken Geschichtsschreibung. Auch in mittelalterlichen und frühneuzeitlichen Quellen taucht der Stammesbegriff immer wieder auf, wo er der Charakterisierung fremder Gruppen, ihrer räumlichen Herkunft, ihrer ,anderen“ Kultur, ihres ,fremden“ Wesens dient. Und Vorstellungen kultureller Zusammengehörigkeit durch Sprache und Werte, aber auch durch Geschichte und regionale Herkunft, die als ethnische ,Zuschreibungskriterien“ immer bis zu einem gewissen Grad „,wandelbar" sind (Elwert I989: 447), prägen auch noch moderne Gesellschaften. Doch betrifft dies bislang nur die kulturelle Produktion von Bildern und Stereotypen. Es ist noch nicht jene nunmehr angeblich wissenschaftlich erhärtete Feststellung, wonach sich Ethnizität an einem „objektiven“ Merkmalskatalog festmachen läßt, der die Zugehörigkeit zu Sprach- und Kulturgemeinschaften an eine genetisch, biologisch und physisch attestierte „Blutsverwandtschaft" bindet. Damit erst sind strikte Grenzen zwischen einem ,eigenen“ Innen und einem ,fremden“ Außen gezogen (Barth I969). Und erst auf dieser scheinbar objektiven Grundlage läßt sich eine ethnische ,Weltanschauung" im wörtlichen Sinne durchsetzen, die über die wissenschaftlichen wie populären Medien vermittelt und zu einem selbstverständlichen Bestandteil, ja zu einem Grundgefühl sozialen Alltagswissens wird: zur ethnischen Identität.

\section{Ethnische Gemeinschaft: Inklusion durch Exklusion}

Dieses ethnische Paradigma baut wesentlich auf zwei gedanklichen Grundlagen auf: zum einen auf jenem romantisch angehauchten Volksbegriff Herders, der zunächst zivilisationskritisch nach kultureller Ursprünglichkeit und Authentizität sucht und diese Eigenschaften im ,einfachen Volk“ und in seinen Überlieferungen zu finden 
glaubt. Schon mit Friedrich Ludwig Jahn aber entwickeln sich daraus verengende Deutungen, die zu einem nationalen „Volkstum“, zum „Deutschtum“ hinführen. Die Vorstellungen von einer „Volksseele“, einer „Volkspersönlichkeit“ schreiben dem eigenen Volk nun eine Individualität zu, die sich im kollektiven „Nationalcharakter“ zu erfüllen scheint. Volk wird immer mehr ,nationales Volk“. Es wird ideologisch aufladbar zu jenem Volks- und Gemeinschaftsbild, das Abstammungs- und Territorialgemeinschaft, Kultur- und Nationsgemeinschaft in eins setzt. Denn auch die Volkskultur erscheint darin einerseits als historisch überlieferte Kultur, die an den mythologischen Ursprüngen anknüpft und deren Ideen lebendig erhält, andererseits als eine ethnische Linie der Überlieferung, als Stammraum und Erbe einer Schicksalsgemeinschaft.

Schon Max Weber charakterisiert diese ,,künstliche“ Art der Entstehung eines ethnischen Gemeinsamkeitsglaubens" als ein obligates und politisch nützliches „Schema der Umdeutung von rationalen Vergesellschaftungen in persönliche Gemeinschaftsbeziehungen": der Mitbürger als Stammesverwandter. Weber sieht darin vor allem eine Strategie der „sozialen Abschließung“ angelegt: das (nationale) „Eigene“" in Blut und Kultur gegen das „Fremde“" (Weber I972: 237f.). Dies bedeutet auch, daß die Überführung ethnischer Motive in nationales Denken dabei hilft, ethnische Fragmentierungen zu überwinden, soziale Gruppen zu integrieren, Gesellschaften oft überhaupt erst mit einem „,nationalen“ Horizont zu versehen. Aber es ist Integration nach innen um den Preis der Abgrenzung nach außen, der Stigmatisierung von sozialen Außenseitern wie anderen Gesellschaften als wesensmäßige und daher bedrohliche „Fremde“, gegen die nur ein waches Gemeinschaftsgefühl schützt: das Prinzip der Inklusion durch Exklusion (Gellner I99I).

Die zweite Grundlage für diese Definition des ethnischen Prinzips legt das naturwissenschaftliche Denken des i9. Jahrhunderts. Dessen biologisches Modell der wissenschaftlichen Einteilung der tierischen und pflanzlichen Welt in Arten, Rassen und Ordnungen scheint geeignet, auch für neue, präzise Vorstellungen menschheitsgeschichtlicher Ordnungssysteme Pate zu stehen. Insbesondere Charles Darwin mit seiner - vielfach verkürzt rezipierten - Lehre vom evolutionären Aufstiegsprinzip von der niederen zur höheren Ordnung und vom biologischen Grundgesetz des survival of the fittest legt den Analogieschluß nahe, daß sich die Vielfalt der physischen und kulturellen Erscheinungsformen menschlichen Lebens ebenfalls in ein Stufenmodell fassen lassen muß. In diesem Modell verkörpert der weiße und zivilisierte Mensch bzw. Mann die „Krone der Schöpfung“, gleichsam als die Verkörperung der wertvollsten genetischen Anlagen und intellektuellen Eigenschaften. Damit lassen sich die Ethnien letztlich 
als Ausdrucksform rassischer Ordnung betrachten und auf der Entwicklungsskala eines universell gedachten Zivilisationsmodells einstufen (Kardorff I99I).

Diese naturwissenschaftliche Anleihe einer Stammes- und Rassenlehre wird zwar nicht von allen in der Volks- und Völkerkunde und selten in aller Konsequenz übernommen. Aber die Perspektive scheint dem zeitgenössischen Denken in „Nationalkulturen“ und „Stämmen“ verwandt und zumindest als generelles Argument einleuchtend genug, um das ethnische Identitätskonzept nachhaltig zu beeinflussen - in der Völkerkunde im Kontext ihrer kolonialen Verwicklungen vielleicht etwas früher, in der Volkskunde etwas später, dann jedoch um so intensiver auf dem Weg zur „Volksgemeinschaft“. Insofern sind beide Fächer an dieser wissenschaftlichen Begründung einer universellen Menschen- und Kulturordnung aktiv beteiligt. Sie tragen wesentlich zur „Ethnisierung“ populärer Vorstellungswelten bei, bestärken die Bilder von kulturell angeblich homogenen Abstammungs- und Volksgemeinschaften und geraten dabei teilweise auch in das Fahrwasser einer physischen Anthropologie. Deren extremste Formen führen dann bis zu einer Rassenanthropologie, die ihren biologischen Rassebegriff durch menschliche Körper- und Skelettvermessungen $\mathrm{zu}$ verifizieren sucht, um dadurch ein anthropometrisches Rassemodell zu erhalten. Auch solche Extreme in Begriffe und Bilder $\mathrm{zu}$ fassen, wissenschaftliche Begründungsinstanz und zugleich populäre Vermittlungsinstanz für ethnische Vorstellungen zu sein, die dadurch den Rang eines universellen „Naturgesetzes" zu erhalten scheinen: dies ist die Last - man möchte auch sagen: Schuld - der ,Volkstumswissenschaften“.

\section{Bilder und Praktiken}

Dabei können wir uns heute nicht mehr auf das lange Zeit bequeme Argument zurückziehen, diese Hypothek sei lediglich von den harten „Rassekundlern“ aufgehäuft worden. Spätestens mit dem Beginn der Aufarbeitung der nationalsozialistischen Fachgeschichte ist vielmehr deutlich geworden, wie sehr daran selbst der scheinbar so harmlose Folklorismus beteiligt war, dessen Bilder von regionalen Trachten und Territorien, von kulturellen Eigenheiten und Eigenarten stets auch ethnisch eingefärbte Mosaiksteine lieferten. Dies bestätigt nicht nur der Blick auf die deutsche Geschichte. Wer die Situation in Jugoslawien zu Anfang der neunziger Jahre beobachtet hat, in der ethnisch-regionale Folklore - gleichsam: Sprach- und Sängerkriege um „Blut und Boden“ - die „ethnischen Säuberungen“ des militärischen Krieges vorbereiten half, kann sich von der Aktualität und der politischen Wirkung solcher „Volks-Kunde“ überzeugen. 
Denn allzu häufig werden dabei regionale Kulturphänomene aus ihrem Zusammenhang herausgelöst, pathetisch überhöht, zum Signum eines „kollektiven Gedächtnisses“ erklärt und zugleich in neue patriotische Erzählungen von „Territorium“, „Ethnie“ und „Nation“ gefaßt. Damit sind sie mit einer neuen sozialen Semantik versehen, die sich strategisch nutzen und fundamentalistisch interpretieren läßt: als ein sendungsbewußter kultureller Alleinvertretungsanspruch gegenüber anderen Meinungen wie Gruppenkulturen, als Zwang zu unbedingter Loyalität gegenüber der beschworenen Gemeinschaft. ${ }^{25}$

Das jugoslawische Beispiel ist sicherlich ein extremes, aber es steht keineswegs allein. Nachdem man noch in den I970er Jahren den Eindruck haben mochte, daß ethnozentrische und nationalistische Konzepte fast schon archaisch anmutende „Auslaufmodelle“ einer Weltanschauung seien, ist nunmehr unübersehbar, daß das Gegenteil eingetreten ist: Eine Welle von neuen regionalistischen und ethnischen Bewegungen hat sich auf eine Sinn- und Identitätssuche begeben, die sich insbesondere nach dem Zusammenbruch des Sozialismus in Osteuropa noch weiter aufgetürmt ${ }^{26}$ und längst globale Wirkung erreicht hat. Wieder wird nach Ursprüngen und Kontinuitäten gesucht, um kulturelle und territoriale Ansprüche zu legitimieren und sie notfalls gewaltsam gegen andere durchzusetzen. Und wieder werden Mythen und Legenden, Kulte und Feste wiederbelebt, um Gefühle zu mobilisieren, Volksstämme zu beschwören, Gemeinschaft zu stiften. Den Charme des Naiven haben solche folkloristischen Beschäftigungen allerdings endgültig verloren. Vor diesem aktuellen Hintergrund bezeichnete der Kulturanthropologe Eric Wolf vor kurzem die Begriffe Rasse, Ethnie und andere fundamentalistische Kulturvorstellungen zu Recht als ,gefährliche Ideen“, die längst (wieder) zu einem ,,ideologischen Kampfmittel in der politischen Auseinandersetzung" geworden sind (Wolf I993: 346).

Nun speisen sich ethnische Zusammengehörigkeitsgefühle keineswegs nur aus Ideologie und Imagination, sie beruhen auch auf konkreten sozialen Praktiken. Neben den traditionellen Formen sozialen Gruppenhandelns hat eben auch dieses „ethnische Paradigma“ durch seine lange Wirkungszeit und dank vielfältiger wissenschaftlicher, politischer wie medialer Vermittlungsstrategien eine hohe Wirkmächtigkeit erzeugt, die uns vielfach fast automatisch ethnisch denken läßt. So sind Bilder ethnischer Identität fester Bestandteil unserer alltagskulturellen Vorstellungswelt geworden, ein selbstverständliches $\mathrm{Zu}-$ ordnungsschema etwa in der Arbeitswelt, in den Medien, in Literatur und Kunst. ${ }^{27}$ Wir haben mit diesem Zuschreibungsprinzip gelernt, unsere Wahrnehmung von Musik oder Mentalität, von Kriminalität oder Migration stets auch auf einer ethnischen Folie zu interpretieren: Die Zeitungsmeldung etwa, die einen ,türkischen“ Jugendli- 
chen als den Täter einer Körperverletzung benennt, setzt bei uns eine reflexhafte Assoziationskette um Begriffe von „Ehre“ oder „Blutrache“ in Gang, die wohl kaum entstehen würde, wäre der Jugendliche „ethnisch“ als Bayer identifiziert.

Sozial anderes Verhalten erscheint in der ethnischen Betrachtungsweise als eine gleichsam systematische Folge kultureller Andersartigkeit. Diese Schematisierung kommt sicherlich auch jener Neigung entgegen (die freilich anthropologisch erst noch genauer zu klären wäre), die Komplexität und Kompliziertheit unserer Welt zu vereinfachen, indem wir deren Erscheinungen zusammenzufassen und zu vereinheitlichen versuchen, indem wir eingrenzen und abgrenzen. Diesem Bedürfnis bietet das ethnische Paradigma Bilder und Stereotype mit scheinbar hoher Plausibilität und Erklärungskraft an, weil soziale Situationen dadurch in der simplen Dichotomie von , innen außen“, ,eigen - fremd“ interpretiert werden können, nach dem simplen Schwarz-Weiß-Modell verfahrend.

Es ist also unnötig, nach mystischen Erklärungen zu suchen, wenn die neue Attraktivität des ethnischen Paradigmas erklärt werden soll. Meist geht es um recht handfeste Interessen: Das ethnische Argument zielt in aller Regel auf Ressourcen- und Gebietsansprüche, auf soziale und kulturelle Machtpositionen, zumal in Regionen, in denen die politischen wie ideologischen Ordnungsgefüge fragil geworden sind. Und die Zahl dieser Regionen scheint weltweit nicht ab-, sondern zuzunehmen. Es ist eine Politik der Differenz und der Macht. Dabei wird im ethnischen Jargon überwiegend kulturell argumentiert, also mit Begriffen von Sprache und Religion, von Mentalität und Authentizität „,kulturalisiert“, weil dadurch starke Bindungskraft nach innen und hohe Legitimationskraft nach außen erzeugt werden können. Vor allem dann, wenn das Gefühl der Bedrohung solcher kulturellen „Grundrechte“ vermittelt wird (Bielefeld I99I).

Nirgendwo bildet sich das gegenwärtig schärfer $a b$ als im Bereich der Migration, wo juristische Barrieren der Einwanderung gegen „Überfremdung“ errichtet werden, selbst in traditionellen Emigrationsländern wie in Italien und Griechenland, die mittlerweile selbst zu Einwanderungsländern geworden sind. Diese ethnisch begründeten Barrieren führen wiederum bei denen, die dennoch ,durchkommen“, zwangsläufig zur Antwort der kulturellen Selbstrepräsentation als ,ethnische Minderheiten“, zur Rückbesinnung auf eigene - vermeintliche? - Traditionen. Solche Auseinandersetzungen um Asylund Staatsbürgerrechte stehen für existentielle gesellschaftliche Erfahrungen: Sie sind nicht nur den Pässen, sondern auch den Menschen tief eingeschrieben. Denen, die juristisch die geforderte Identität besitzen, wie denen, die sie - allerdings nicht um den Preis der Selbstaufgabe - erwerben wollen. Beiden ist damit die Beachtung jenes 
schon überholt geglaubten Abstammungsprinzips wieder zu einer Überlebensfrage gemacht worden. Dies gilt auch für "klassische“ Einwanderungsländer wie Frankreich, in denen heute gerne vergessen wird, daß die Anwerbung ausländischer Arbeitskräfte schon seit dem Ende des I9. Jahrhunderts eine bewußte politische Entscheidung war (Augé I995). Der Historiker Gérard Noiriel hat rekonstruiert, wie damals explizit um „Einwanderer“ geworben wurde, da große einheimische Bevölkerungsgruppen die Industriearbeit ablehnten (Noiriel I988). In diesen Jahren schien sich die Grande Nation noch nicht in ihrer Identität bedroht zu fühlen - allerdings kam diese Einwanderung auch noch nicht wie heute zu über fünfzig Prozent aus den „,dunklen“ nordafrikanischen Ländern ...

Für eine Ethnologie, die sich ihrer fachgeschichtlichen wie ihrer gesellschaftswissenschaftlichen Verpflichtung bewußt sein will, hat der Begriff der Ethnizität jedenfalls seine Unschuld verloren. Wenn jetzt Kulturwissenschaftler davon sprechen, daß nach dem Ende der ,großen“ sozialen und nationalen Erzählungen nun „,ethnische Differenz zur Quelle von Identität" werde, und wenn sie dies nicht kritisch beobachten, sondern ihrerseits die ,ethnische Revolution als eine globale Alternative zur Krise der alten Ordnung und zur Herausforderung durch die neue ,postmoderne" Welt" propagieren, gar als die ,äußerste Form“ der Abwehr all jener Uniformierungs- und Standardisierungstendenzen unserer kulturellen Identität (Durando I993), dann werden kritisches Denken wie geschichtliche Erfahrung auf den Kopf gestellt. Es wird - noch zurückhaltend formuliert wissenschaftliche Naivität zu einer gesellschaftlichen Gefahr. Denn dieses ethnische Argument erwies sich fast immer als ein extrem aggressives Identitätskonzept, das sich beinahe beliebig in rassistische, nationalistische und hegemoniale Strategien einpassen ließ. Das wissen wir inzwischen, aber offenbar haben wir dies nicht laut genug gesagt, um solche wissenschaftlichen „Wiederentdeckungen“ zu verhindern.

In diesem Versäumnis scheinen sich eigene Unsicherheiten darüber auszudrücken, ob die Rede von der ethnischen Identität als „Konstruktion“ nicht eine lediglich intellektuelle Denkfigur sei, der im Alltag ein populäres Denkprinzip gegenüberstehe, das sich unbekümmert auf ethnische „Wirklichkeiten“ beruft. Es ist sicherlich kein Zufall, daß wir bei solchen Gegenargumenten meist sofort die generelle Notwendigkeit von Gruppenidentitäten einräumen, die in einer "world in transition" als Halt und Orientierung unverzichtbar seien. Wie solche Identitätsvorstellungen dann freilich ohne ethnische Komponenten und Differenz aussehen sollen, wird von uns meist nicht näher beschrieben. Denn mit „Gemeinschaftsvorstellungen“ tun wir uns - aus guten wissenschafts- wie gesellschaftsge- 
schichtlichen Gründen - ohnehin schwer. Die Frage, wie sich soziale Bindungen innerhalb der Gesellschaft auch symbolisch und emotional gestalten, ob dies nicht immer zunächst über die Konstruktion eines „Anderen“ geschieht, bevor man wahrnehmen kann, „daß das Eigene auch Aspekte des Fremden birgt" (Schiffauer I996: 30) diese Frage steht in gewisser Weise offenbar unter Ideologieverdacht bzw. gegen die Regeln der Political Correctness. Nun kommt der einst geworfene ethnische Bumerang jedoch zurück, und er trifft ins Zentrum der Fachidentität: Der Frage nach dem Umgang mit einem ethnos, das nicht mehr in grauer Geschichte, sondern mitten in der Gegenwart zu verorten ist, kann eine Europäische Ethnologie nicht länger ausweichen. Die - nicht immer glücklich geführte - Diskussion um „multikulturelle“ Konzepte gesellschaftlichen Lebens zwingt dazu, wissenschaftlich wie politisch Farbe zu bekennen. ${ }^{28}$

Wenn Ethnizität also von den „Leidenschaften der kollektiven Identität" handelt, wie Clifford Geertz einmal formulierte, dann sollte unser Fach zu zeigen versuchen, wie diese Leidenschaft $\mathrm{zu}-$ nächst in Europa erlernt wurde und welche unauslöschliche Spur sie in unserer Geschichte hinterlassen hat. ${ }^{29}$ Und umgekehrt wäre zu verdeutlichen, wie sich andere, weniger schmerzhafte Formen der Erfahrung von Zusammengehörigkeit entwickelt haben - Modelle sozialer Loyalität, die im Schatten solcher dominanten Konzepte wie Nation und Ethnie geblieben sind. Das Fach hat in seiner Geschichte dazu beigetragen, derlei Alternativen unsicher und unsichtbar zu machen. Eigene Blindheiten zu korrigieren und dabei das ethnische Paradigma im Sinne des Verursacherprinzips gleich selbst mit zu entsorgen, es in konkreten ethnographischen Studien als kulturelle Denkfigur $\mathrm{zu}$ entideologisieren und zu entdramatisieren scheint mir heute eine lohnende Herausforderung zu sein.

\section{Verortungen: Schicht und Geschlecht}

Mit der Frage nach der sozialen und geschlechtlichen Ortsbestimmung innerhalb des gesellschaftlichen Gefüges sind zwei Grundvorstellungen benannt, die das Thema Identität in vieler Hinsicht weiterführen bzw. es genauer fassen. Jenes generelle „Wer bin ich?“ erhält dabei die Zusatzfrage: „Wo stehe ich, wozu gehöre ich?““. Diese Fragen und die Antworten darauf geben zugleich Aufschlüsse darüber, wie wir, indem wir unsere eigene Position zu beschreiben versuchen, eine allgemeine „Ordnung der Gesellschaft" wahrnehmen.

Die Kategorien Schicht und Geschlecht verbinden zunächst einige gemeinsame Eigenschaften: Beides sind Beschreibungsdimensionen individueller Identität, die sich allerdings nur aus der Beziehung zu 